\title{
ENTREVISTA AL DR. PEDRO GRADOS SMITH
}

Vicepresidente de Wealth Management de Scotiabank Perú, Miembro del Directorio de Profuturo AFP y Miembro del Comité Consultivo de la Carrera de Administración y Finanzas, Facultad de Negocios, UPC.

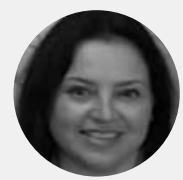

\section{MARÍA DEL CARMEN ARRIETA}

- Profesora de Teoría Microeconómica

Carrera de Administración y Finanzas

Facultad de Negocios, UPC

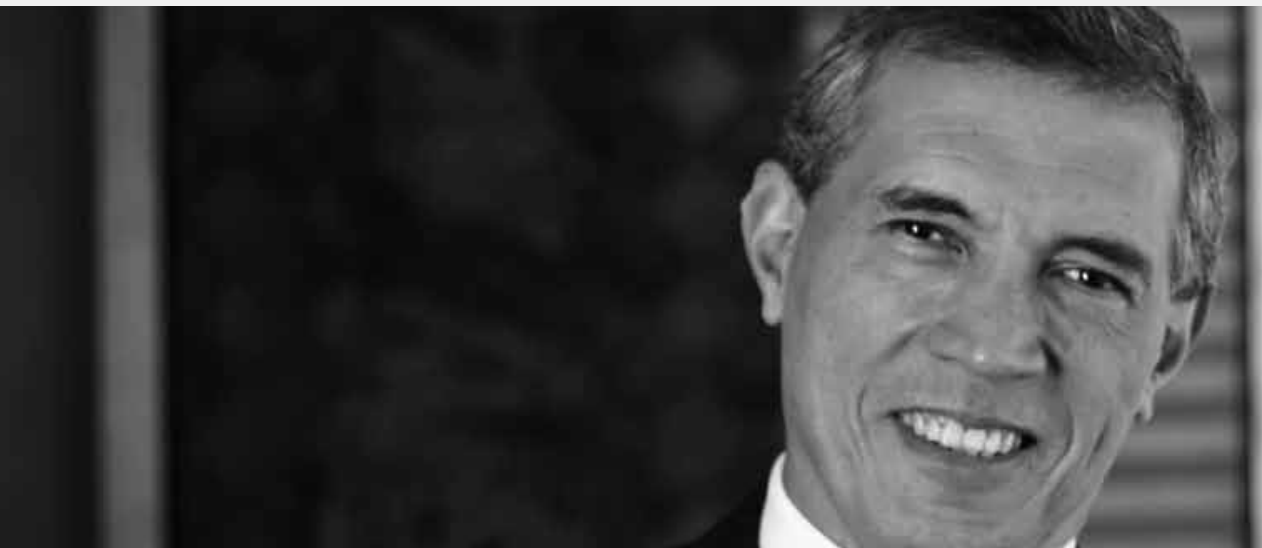

\section{ESTUDIOS}

Estudios de Doctorado Internacional en Administración y Dirección de Empresas en la Universidad Politécnicade Cataluña de Barcelona, España. Maestría en Banca y Finanzas y Economista graduado en la Universidad de Lima. Adicionalmente, cuenta con una especialización en Contabilidad y Finanzas de la Universidad ESAN.

\section{TRAYECTORIA PROFESIONAL}

Gerente General Adjunto y Gerente de Inversiones de Profuturo AFP. Anteriormente fue Superintendente Adjunto de Banca y Micro finanzas en la Superintendencia de Banca, Seguros y AFP. Gerente de Riesgos de la SBS y ocupó las Gerencias de Finanzas, Control Interno y de Riesgo de Mercado y Economía en el Banco Santander Central Hispano S.A. Perú. Asimismo, fue Gerente General de Santander Sociedad Agente de Bolsa y Gerente de Desarrollo de la Bolsa de Valores de Lima. Ex Director de la Bolsa de Valores de Lima y de CAVALI. Es profesor del PAD de La Universidad de Piura y de la Universidad Peruana de Ciencias Aplicadas (UPC) en cursos de Finanzas y Economía. Ha sido profesor de la Escuela Diplomática del Perú y de la Universidad de Lima, Ex Presidente del "Comité de PROINVERSIÓN de Saneamiento y Proyectos del Estado"y Ex Director de La Corporación Financiera de Desarrollo S.A. - COFIDE y de NCF Inversiones. Adicionalmente, ha sido Consultor de la Corporación Financiera Internacional IFC Consultor de USAID, y consultor de La Bolsa de Valores de Lima.
¿Representan las AFPs en el Perú una industria financiera oligopolizada?

La industria de AFP's, al igual que muchas otras industrias en mercados emergentes, tiene características oligopólicas; si hablamos de la industria de distribución de energía en Lima, hay dos empresas que la distribuyen: Luz del Sur y Edelnor en Lima; si hablamos de la industria telefónica hay cuatro operadores telefónicos: Telefónica, Claro, Entel y los vietnamitas, hay muchas industrias oligopólicas. En la industria de AFPs, son básicamente cuatro operadores, tres de ellos con historia larga, uno con historia corta, y el problema no es que exista alguna barrera, no es que exista una prohibición para ingresar, sino que hay una barrera natural de ingreso que genera una industria con características oligopólicas: el periodo para recuperar la inversión, ni siquiera para recuperar la inversión, sino el periodo para pasar a flujo de caja positivo mensual, va entre los tres y los seis años. Cualquier inversionista que quiera entrar tiene que estar dispuesto a perder dinero entre tres y seis años, y es lo que le está pasando a Hábitat, Hábitat está perdiendo aproximadamente entre millón y millón y medio de soles mensuales, por lo tanto es una apuesta de largo plazo. 


\section{¿Al ser pocos operadores, dónde se da la competencia?}

No hay competencia de nuevos afiliados, porque los nuevos afiliados van exclusivamente al que ganó licitación. La regulación del mercado, al subastar a todos los nuevos clientes cada dos años, está tratando precisamente de romper con cualquier comportamiento oligopólico y eso ha conducido a una reducción de las comisiones. El tema es bastante complejo porque ha cambiado la forma de cobrar comisiones: los que nos hemos quedado con la comisión por flujo o por sueldo, la cual a lo largo del tiempo desaparecerá, y el nuevo esquema, que va a ser cobrar por activos por administración, pero es todo un proceso para llegar a ese nuevo esquema. En conclusión, es una industria con características oligopólicas al ser pocos operadores, esto se debe, básicamente, a las altas barreras de ingreso que existen por el costo, el periodo de recuperación del dinero, sin embargo los beneficios que ha generado desde el punto de vista de fondos administrados, de mayores pensiones y de no depender del estado, son significativamente mayores que estas críticas vinculadas a las características de industria oligopólica.

\section{De tener poder de mercado, las comisiones y seguros varían estadísticamente, ¿por qué las diferencias?}

En la parte de seguros ya se rompió cualquier tipo de comportamiento dentro de una estructura oligopólica porque, cuando tú pagas en la industria de pensiones, tienes dos costos: la comisión, sea esta por flujo o sea mixta, que es la comisión que te cobra la AFP por gestionar los activos, y la prima que pagas para el seguro de invalidez y sobrevivencia, es decir, se te pasa algo durante tu vida activa estás cubierto por un seguro de invalidez si no puedes trabajar y por un seguro de sobrevivencia si es que por alguna razón muere el afiliado; los beneficiarios recibirían una pensión.

La prima se subasta para la industria en su conjunto y pueden participar todas las compañías de seguros registradas en el Perú. El proceso implica una separación en siete segmentos y al final se cobra una prima promedio en función a estos siete segmentos y por lo tanto la prima es exactamente la misma para el universo de los miembros del sistema privado de pensiones, por lo tanto el único factor de competencia cuantitativo hoy en día es el precio que cobra cada AFP. Si hablamos de los atributos de la industria hay básicamente cuatro atributos: un atributo, que es generalmente el que está primero es el servicio; por servicio entenderíamos información sobre todo el sistema de aporte y jubilación y facilidades en el proceso mismo de jubilación, y podemos incluir ahí pagos a las personas que han obtenido una pensión de invalidez, de sobrevivencia o de jubilación. La segunda variable es generalmente rentabilidad, y hoy día la rentabilidad es sumamente importante, porque de los 123,000 millones de soles administrados aproximadamente entre 50\% y $60 \%$ es rentabilidad generada desde el origen del Sistema de AFP. El tercer atributo es precio y en la medida que iremos a cobrar por activos bajo administración, que esa es la reforma, el precio va a estar incorporado dentro de la rentabilidad, es decir tu rentabilidad va a ser libre de "polvo y paja", o libre de cualquier costo transaccional que se de en la gestión, y eso va a ser mucho más transparente de lo que es hoy día. El cuarto atributo es solvencia. Por lo tanto, los atributos para que alguien entre al sistema tienen que ver con servicios, con rentabilidad, con precios por servicio, y con solvencia.

¿Por qué considera usted que la industria de AFPs en el Perú tiene tantos detractores?

\section{...MUCHAS EMPRESAS ESTÁN OBSESIONADAS POR LOS RESULTADOS DEL AÑO Y SE OLVIDAN QUE A VECES ES IMPORTANTE SACRIFICAR EL RESULTADO DEL AÑO PENSANDO EN INVERSIONES Y EN UNA RECUPERACIÓN DE MÁS LARGO PLAZO DE ESA INVERSIÓN...}

Bueno, hay muchas razones, primero está la teoría del comportamiento económico que tiene que ver, según los teóricos partidarios de esta corriente de pensamiento, con que el ser humano suele pensar más en el corto plazo que en el largo plazo; esa racionalidad te lleva a pensar más en beneficios de corto plazo que en los de largo plazo, muchas empresas están obsesionadas por los resultados del año y se olvidan que a veces es importante sacrificar el resultado del año pensando en inversiones y en una recuperación de más largo plazo de esa inversión, en un mayor valor de la empresa. De igual forma, muchos individuos, muchos seres humanos piensan más en el hoy, hoy tengo que aportar el 10\% de mi remuneración o entre el 12\% y 13\% incluyendo primas y comisiones para cuando me jubile dentro de 10 , 20, 30 o 40 años, y ahí hay un tema, no necesariamente somos conscientes del largo plazo según los teóricos de la economía del comportamiento. Segundo, es muy fácil criticar una industria que ofrece beneficios en el largo plazo. Tercero, hay una responsabilidad de la propia industria de no haber difundido todos los beneficios de la misma, lo cual implica invertir en talleres en las universidades, en las empresas, en publicidad educativa, etc. Un ejemplo de lo beneficioso del sistema: hoy en día, la pensión promedio en el sistema privado es 1,100 soles (aproximadamente) versus 700 soles (aproximadamente) en el sistema público; pero además esta es una pensión que depende $0 \%$ del presupuesto del estado, por lo tanto, son 1,100 soles auto sostenibles versus una pensión pública que no es necesariamente auto sostenible. 


\section{¿Qué opina de las últimas declaraciones en Chile del Nobel de Economía Joseph Stiglitz que le colocó la lápida al sistema de AFPs?}

Cualquier nobel, y cualquier economista, tiene una visión del mundo con cierto sesgo; Joseph Stiglitz ahora está más en la crítica del sistema de economía del mercado en general; aquí si mal no recuerdo, fue Winston Churchill, quien dijo que la democracia, y la economía de mercado, no son lo mejor, pero no se ha descubierto nada que lo supere. Entonces hay muchas formas de criticar, pero finalmente creo que en el tema de las pensiones la realidad en el Perú es que el Sistema de AFP muestra mejores pensiones que en el sistema público; y además estas pensiones han liberado recursos en el sector público para otras funciones, por lo tanto sí es un sistema solidario en el sentido que al liberar recursos y no depender del estado, el gobierno tiene recursos para por ejemplo generar lo que se conoce como: el pilar solidario, es decir recursos para aque que no pudo generar una pensión, y eso es pensión 65, y la pensión para los discapacitados que acaba de salir. Nadie dice que no sea necesaria pero finalmente es un subsidio de toda la población a través del presupuesto del estado para sectores que por alguna u otra razón no han sido capaces de generar su propia atención, eso también creo que es un factor importante.

\section{¿Cómo afectará al rendimiento de las AFPs la última caída de la bolsa China? ¿la economía China en general? Ante eso: ¿qué medidas están tomando o tomarán las AFPs?}

En general lo que está pasando en la economía mundial afecta a los activos financieros en el mundo, y por lo tanto afecta a los activos financieros en el Perú; la ren- tabilidad de las AFPs en términos de soles, ha sido positiva, básicamente se mantiene positiva en el fondo uno y dos, en el tres probablemente no, al día jueves todas son positivas, al día de hoy, no tenemos la data oficial del día viernes, del lunes y martes (esta entrevista se realizó el día miércoles 26 de agosto) y tal vez no lo sean en el tres, pero el uno y el dos sí lo son, me refiero en soles. Pero básicamente, y sale un artículo hoy día en Gestión, un porcentaje importante de la cartera está dolarizada y eso ha protegido a los fondos.

\section{¿Por lo tanto se va a ver afectada?}

Sí, al final el valor de todos los activos se ven afectados por lo que ocurre en el mundo.

\section{Para muchos analistas estamos a puertas de otra crisis económica mundial ¿Qué opina al respecto?}

A ver, si hablamos de los últimos 100 años podremos decir que las dos peores crisis han sido la de 1929 financieramente hablando, y probablemente la del 2008; como crisis financieras, crisis muy duras; la del 29 largamente peor que la del 2008, yo diría que estamos en una situación de incertidumbre porque se han aplicado políticas monetarias y fiscales para evitar una depresión como la de los años 30, que en lo inmediato como resultado, han dado resultados positivos, pero que en lo que viene requiere un manejo fino de ajustes macroeconómicos a nivel mundial de alguna $u$ otra forma. En términos generales, lo que ha ocurrido es un gran aporte fiscal en la economía como la norteamericana o la europea y políticas monetarias que han tratado de evitar la recesión, ahora lo importante es manejar esta situación macro con mucho juicio porque, nunca se ha emitido la cantidad de dinero que se ha emitido en los últimos siete años. Por lo tanto, hay que retirar esa cantidad de dinero, ni tan rápido como para irnos a una recesión, ni tan despacio que pueda generar inflación. La verdad es que hasta hoy no se ha generado inflación producto de estas emisiones, hay que ser muy pragmático en la gestión hacia adelante, la recuperación de EEUU es relativamente sólida, creo que el caso chino es un caso complejo, y lo mejor para una economía como la peruana es diversificarse y mantener sólidos fundamentos macro.

\section{¿De qué manera estamos cubriéndonos ante términos de intercambio menos favorables?}

Ahí lo que hay que hacer, y hay una larga tarea por realizar considerando que somos todavía un país que dependemos en un porcentaje importante de commodities, materias primas, es generar productos con mayor valor agregado, hacer crecer las llamadas exportaciones no tradicionales, donde sí hay resultados interesantes por ejemplo es en el sector agropecuario, somos importantes exportadores de espárragos, de uvas, y eso no es producto que haya exportado Perú tradicionalmente; además, el sector metal-mecánico también ha crecido sobre todo a base del crecimiento minero local, también nos hemos expandido a nivel internacional; tercero, hay empresas a las que el crecimiento de la economía peruana y su apertura les ha permitido tener una visión más de tipo multilatina Hay casos a diferentes niveles, Graña se ha expandido en la región, Ajé Group se ha expandido en el mundo, Yanbal también ha tenido un crecimiento interesante, y yo creo que ahora lo que hay que ver es cómo enfrentar términos de intercambio negativos, hay que diversificar mercados y en eso hemos avanzado, pero sobretodo hay que diversificar productos y hay que pragmáticamente generar incentivos con retorno a exportaciones no tradicionales y a exportaciones con un mayor nivel de valor agregado pero no artificialmente sino algo que sea sostenible, no hay fórmula mágica ahí, tu das un subsidio y pueden crecer pero quitas el subsidio y debería ser autosostenible, y esa es una variable bastante compleja. Pero bueno, los términos de intercambio nos están afectando, yo creo que el súper ciclo de materias primas que tuvimos no va a regresar a corto plazo.

\section{LO MEJOR PARA UNA ECONOMÍA COMO LA PERUANA ES DIVERSIFICARSE Y MANTENER SÓLIDOS FUNDAMENTOS MACRO.}


¿Qué perspectivas ve para lo que queda del 2015 y lo que viene del 2016?

En el mundo, creo que si no se da un tipo de catástrofe económica en China, se van a ir solucionando los problemas poco a poco, pero todavía con un tema de incertidumbre en los próximos meses. En América Latina es complejo, porque la región es compleja, porque hay países que están muy golpeados y a los que no les veo solución inmediata guardando las diferencias: Venezuela y Ecuador son países complicados, Venezuela más, Ecuador un poco menos, Argentina también, por qué, porque son países que no han sacado provecho en la búsqueda de una mayor o mejor estabilidad macroeconómica producto del ciclo de crecimiento impresionante en los precios de las materias primas y hoy día enfrentan déficits fiscales y déficits de cuenta corriente significativos, además de bajas reservas. Sin embargo, países como los de la Alianza del Pacifico, México, Chile, Perú y Colombia, tienen fundamentos macro más sólidos y van a depender de un manejo pragmático de las variables macro por parte de las políticas del gobierno, pero siempre a lo que tienen que tender es a tener empresas y personas más competitivas y eso por parte de las personas implica que estén mejor formadas, mayor capital humano producto de una política educativa a largo plazo; en eso también el Perú ha avanzado, aunque los resultados no los vea este gobierno, probablemente se vean todavía en dos, tres, cuatro, cinco o diez años si se sigue por ese camino, eso es lo fundamental, ser más competitivo, no tener miedo a competir.

\section{¿Qué significa la Alianza del Pacifico para las AFPs?}

Es algo muy importante pero tienen que uniformizarse las regulaciones, creo que es una posibilidad de crecimiento de la economía producto de toda la teoría de integración, amplías mercado, puedes llevar tus productos, etc. Pero además las AFPs pueden invertir en otros mercados, ahí sin embargo requeriríamos que se genere un límite especial de inversiones en la Alianza del Pacifico; hoy día tienes hasta 50\% del portafolio que puedes invertir en el exterior sin discriminar ningún tipo de país, probablemente lo que sería interesante es que se ponga un límite de hasta 100\% para Perú o países de la Alianza del Pacifico, lo cual te genere una integración, y para el resto si quieres mantén el 50\%.

\section{¿En su opinión se verían afectadas las AFPs por un aumento de la remuneración mínima?}

Esta es una discusión teórico-práctica y nada política, es muy fácil decirle a una persona que gana el sueldo mínimo: "vamos a aumentar el sueldo mínimo por ley" y la persona si no pierde el trabajo debe ganar 750, 800, 850 0 1,000 soles, claro, esa persona se va a sentir mejor, un aumento en el sueldo mínimo beneficia en el corto plazo a las AFPs porque si tú ves la masa salarial, hay una masa salarial importante cuya remuneración mensual está en función al sueldo mínimo, sea este uno, dos, tres o "n" veces el sueldo mínimo, al subir el sueldo mínimo suben los ingresos de las AFPs, pero es una ilusión porque lo que tu requieres, y esto es en el tema de mercado, es una remuneración, un costo laboral bastante flexible y que quede en función de la productividad, es fácil de decirlo, difícil de implementarlo y difícil de explicarlo desde una perspectiva política y para el público en general. El trabajo es un servicio que se demanda y si tú pones algo mínimo que está por encima de la productividad estás distorsionando la asignación de ese recurso escaso, yo diría en el corto plazo un aumento en el sueldo mínimo va a beneficiar a las AFPs en sus ingresos, en el largo plazo, al perjudicarse la economía, dependiendo de cuál sea el nivel de este sueldo mínimo, puede perjudicar también el crecimiento de las empresas peruanas y el empleo y por ahí se pueden ver afectadas las AFPs.

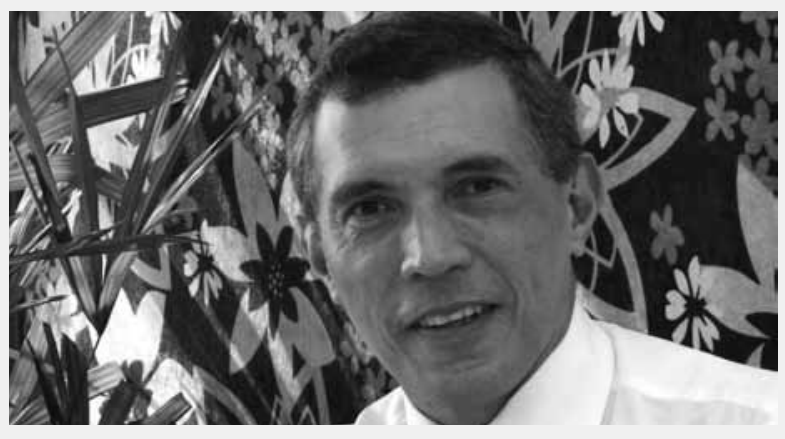

\section{¿Cuál considera Ud. que es el objetivo de la SBS al tener dos alternativas para el cobro de las comisiones?}

En el largo plazo la idea es quedarte con uno solo que es cobrar por activos bajo administración lo cual es más transparente, además es una práctica internacional que implica un mantenimiento directo de tu rentabilidad libre de costos; como se cobra en el Perú, en el esquema de flujo es difícil saber cuál es tu rentabilidad después del pago de comisiones porque depende de cada uno, caso por caso, si has aportado o no has aportado, yo creo que hay un beneficio directo y la forma como lo han hecho es simplemente un periodo de tiempo para pasar de un esquema de flujo a un esquema de saldos. 


\section{¿Están las AFPs excesivamente reguladas en el Perú en detrimento de su crecimiento? ¿Qué modificaría y por qué?}

Están reguladas y probablemente es la industria financiera más regulada, más que los bancos; por otro lado hay que buscar los equilibrios porque al ser una industria con características oligopólicas lo importante es que la regulación sea la adecuada y creo que ahí se está avanzando, por ejemplo nos van a dar más libertad en la medida que tengamos los equipos de personas y de sistemas necesarios para operar en lo que se refiere a la gestión de inversiones; pero, y es una opinión, claramente la industria de AFPs tiene que ser regulada; lo que pasa es que tiene que ser regulada por un sector público eficiente, con capital humano sofisticado, etc.; y por qué regulada me dirán algunos, porque también está regulado el aporte a las AFPs, uno está obligado a pagar una cantidad mensual, ya sea al sistema público o al privado para generar su pensión, en la medida que te obligan, el Estado tiene un rol que es: tratar de que esa obligación sea gestionada de la mejor manera posible y dentro de una industria con pocos actores, yo creo que pensar en lo otro es tener una visión distorsionada, no es un mercado de competencia perfecta, no nos engañemos tampoco, no existen muchos compradores ni muchos vendedores, está bien.

\section{¿Es de la opinión de que el ahorro previsional público debe eliminarse? ¿Por qué?}

En el largo plazo sí, porque tú tienes tres pilares en los sistemas previsionales, el pilar solidario, es decir para aquel que no contribuyó lo necesario para generar una pensión, pensando en evitar la pobreza en el adulto mayor y pensando en el devolverle lo que le dio a la sociedad, debe otorgarle una pensión mínima, eso va a depender de todos, porque esto se financia con los impuestos a través del tesoro, si se lograra que todos generáramos aportes y por lo tanto una pensión aparte de la del sector público, yo te diría que no se requeriría una pensión solidaria pero eso es ya como la situación perfecta y hay un largo camino para llegar, por lo tanto sí se requiere un pilar solidario; el segundo es el pilar obligatorio, en el Perú tiene dos esquemas, el pilar obligatorio para las AFPs y el pilar obligatorio para la ONP, algunos casos particulares como la Caja Militar y Policial, tú decides en cuál estás, por qué digo que el público debería eliminarse, porque al final tal y como está estructurado hoy día el público te genera una pensión mínima, cosa que no te genera el sector privado, pero esa pensión mínima la verdad debería ser ofrecida a todos porque la pensión mínima sale del presupuesto público y bajo el mismo concepto en la medida que todos seamos capaces de aportar para la generación de una pensión, debería haber una pensión mínima que sea aportada por el Estado, que esté mucho más vinculada a un contrato social, a esta idea de que ningún adulto mayor debe ser pobre, más aun si trabajó durante toda su etapa activa. La competencia entre un sistema que te genera una pensión mínima subsidiada es un perjuicio para el afiliado de alta renta aunque casi no hay ninguno en la ONP, todos están en las AFPs, y un sistema que no te genera ninguna pensión mínima que es el sistema de AFPs realmente hay un tratamiento no homogéneo que distorsiona la toma de decisiones de las personas; yo creo que lo ideal sería generar un proceso de transición que puede ser de largo aliento cinco, diez, quince años, no lo sé, para tratar de evitar problemas como los de Europa, sobre todo Grecia, España, Italia, etc., donde las pensiones no han logrado sostenerse y finalmente terminan en una disminución de la jubilación o en un incremento del aporte, sí deberíamos de pensar en la eliminación de lo que es el sistema público. Finalmente, el tercer pilar son los aportes voluntarios para aquellos que desean incrementar sus pensiones.

\section{¿De qué manera contribuye el sistema de AFPs al desarrollo del país?}

Primero, porque resuelve en parte el problema de las pensiones, lo que no resuelve, y es un problema que va más allá del sistema previsional privado, es la cobertura debido al alto nivel de informalidad, donde la población económicamente activa no está en el sector formal. El porcentaje de trabajadores que no están en el sector formal tampoco está generando su pensión. La cobertura hay que resolverla de otra manera, para que de una u otra forma en el largo plazo sólo quede el privado, en los próximos cinco años hay que mantener los dos, hay que generar un proceso de cambio y el aporte voluntario para aquellos que quieren siempre incrementar su fondo de pensión. Las AFPs te resuelven un tema de la propia pensión pero además permiten desarrollar un tema de mercados de capitales de manera significativa, son los principales inversionistas institucionales en el Perú y por lo tanto los principales inversores en el mercado de valores a corto, mediano, y largo plazo, adquieren bonos a diez, quince, veinte años a más, vinculados muchos de ellos a infraestructura.

\section{¿Cuál ha sido el rendimiento promedio anual histórico del fondo tipo II?}

El rendimiento promedio anual en el fondo tipo II es alrededor de $12 \%$ nominal en soles, sin considerar el efecto de la comisión, lo cual te podría generar entre 7\% y 9\% si se le considera.

\section{¿Cuál es la perspectiva de su rentabilidad anual a largo plazo?}

La perspectiva es que se mantenga en el largo plazo, pero creo que el $12 \%$ promedio anual han sido resultados muy buenos que deberían ir más hacia un 10\% u 8\%. 


\section{¿Por qué Profuturo ha tenido tanto éxito en la administración del fondo tipo III en el último año?}

Yo diría que la clave nuevamente está en el capital humano y en el proceso de inversiones, se ha contratado un capital humano de primer nivel en términos internacionales que está gestionando acá 10,000 millones de dólares, podría estar gestionando fondos de pensiones en Nueva York o Londres; un equipo de inversiones muy sólido académicamente, con maestrías y doctorados en el exterior en Finanzas o en Economía, y con experiencia en el mercado, yo diría que esa es la clave, un proceso de inversiones muy disciplinado y una gerencia de riesgos muy profesional.

\section{¿Por qué solo tres tipos de fondos?}

Bueno, va a surgir un cuarto tipo que es el fondo cero, yo creo que para una primera etapa está bien, después se puede dar un esquema como el chileno en el que son cinco tipos, y después quizás hasta un esquema más abierto.

Luego de quince años de implementado el límite de inversiones en el exterior, ¿Cuál es el balance de los resultados de las inversiones en el exterior versus las inversiones domésticas?

De no haberse implementado las inversiones en el exterior, los últimos tres años hubieran sido catastróficos para la industria de AFPs, lo que nos salvó son las bolsas externas, el año pasado casi hubo un comportamiento opuesto en ambos casos.

\section{¿Profuturo tiene alguna propuesta para los límites de inversiones en el exterior a largo plazo?}

Sí, lo ideal es que se te permita gestionar un portafolio sin ningún límite, pero entendemos que estos límites son necesarios tanto desde una perspectiva de un mercado monetario o cambiario, políticas del Banco Central, como de una perspectiva de identificación con el país, generación de efecto multiplicador con un ciclo de crecimiento de largo plazo, pero lo ideal es que tú puedas generar tu portafolio sin mayores limitaciones.

\section{Con respecto a los aportes voluntarios, ¿qué recomendaciones daría usted a los afiliados?}

La verdad es que en el Perú, a diferencia de Colombia y Chile, los aportes voluntarios no son significativos, y no lo son por dos razones principales, primero porque no están exonerados del impuesto a la renta; y segundo, porque en el Perú, a diferencia de Colombia y Chile, no existe un límite para el aporte obligatorio. Por lo tanto, en Colombia y Chile si tú quieres mantener una mayor tasa de reemplazo, es decir tu pensión con respecto a tu ultima remuneración o a un promedio de las últimas remuneraciones, es más importante un aporte voluntario. dígase Essalud. 